\title{
Actividad de cobre sobre bacilos gramnegativos multi-resistentes aislados en hospitales chilenos
}

\author{
Tomás Kappes, Mariana Domínguez, Helia Bello, Sergio Mella, Gisela Riedel y Gerardo González-Rocha
}

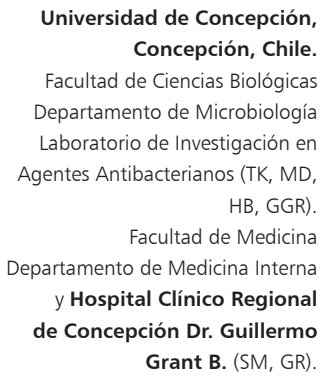

No existen conflictos de interés. Fuente de financiamiento: Facultad de Ciencias Biológicas. Universidad de Concepción

Recibido: 7 de mayo de 2012 Aceptado: 6 de noviembre de 2012

Correspondencia a: Gerardo González-Rocha. ggonzal@udec.c

\section{Copper activity against multiresistant Gram negative bacilli isolated from Chilean hospitals}

Introduction: Multiresistant nosocomial pathogens, especially Gram-negative bacilli (GNB), are a serious problem for public health systems worldwide. Due to their antimicrobial properties, copper alloys have been suggested as an alternative for the control of bacterial burden in surfaces in hospital environment. However, antibiotic multiresistance and copper resistance could be associated in GNB, and there is evidence that both kind of resistance genes (antibiotic and copper) can be located on the same genetic structures. For this reason antibiotic-multiresistant strains could survive in the presence of copper, selecting for bacterial phenotypes resistant to both antibacterial agents. Aim: To evaluate antibacterial activity of copper against nosocomial extended-spectrum $\beta$-lactamases (ESBL) (+) and ESBL (-) GNB, and carbapenems resistant or susceptible strains. Material and Method: This study included 390 strains of GNB isolated from Chilean hospitals: Acinetobacter baumannii and Pseudomonas aeruginosa resistant $\left(\mathrm{CAR}^{\mathrm{R}}\right)$ and susceptible $\left(\mathrm{CAR}^{\mathrm{S}}\right)$ to carbapenem antibiotics, and Klebsiella pneumoniae and Escherichia coli producers and non-producers of ESBL. Susceptibility levels to cupric sulphate were determined by agar dilution method and statistical analysis were used to determine the significance of the differences in the copper tolerance levels between the strains groups. Results: Statistically superior copper tolerance levels were found in the $\mathrm{CAR}^{\mathrm{R}}$ and ESBL producing strains of $A$. baumannii and $K$. pneumoniae, in relation with the $\mathrm{CAR}^{\mathrm{S}}$ and ESBL not-producing strains. Conclusion: A relation between a diminished susceptibility to ionic copper and to recent generation antimicrobial agents was observed in K. pneumoniae y A. baumannii strains.

Key words: Copper tolerance, antibiotic resistance, nosocomial infections.

Palabras clave: Tolerancia a cobre, resistencia antimicrobianos, infecciones nosocomiales.

\section{Introducción}

A ctualmente las infecciones asociadas a la atención de salud (IAAS) constituyen un grave problema de salud pública en el mundo debido al aumento en las tasas de mortalidad, morbilidad y tiempo de hospitalización de los pacientes afectados por ellas ${ }^{1}$. Esto se traduce en un drástico incremento en el costo directo del tratamiento de los pacientes. Se estima que anualmente en Estados Unidos de América se producen 2 millones de IAAS con un costo de más de US\$ 4.500 millones $^{2}$, en Inglaterra se registran 300.000 IAAS al año con un costo aproximado de US\$ 1.600 millones $^{3}$, mientras que en Chile, se informa de 70.000 IAAS por año, aumentando la hospitalización en un promedio de 10 días, lo que tiene un costo de US\$ 70 millones para el sistema público de salud $^{2}$. No obstante, la magnitud económica real de esta problemática es aún mayor debido a los costos indirectos que estas infecciones producen y que no son considerados por la mayoría de los estudios.

Entre los agentes etiológicos asociados a IAAS, especialmente en Latinoamérica y Chile, destacan ciertos bacilos gramnegativos que constituyen un subconjunto de alta importancia clínica ${ }^{4}$. En nuestro país son los principales agentes etiológicos de IAAS del tracto urinario y neumonías asociadas a ventilación mecánica en pacientes adultos y pediátricos ${ }^{5}$. Uno de los mayores problemas asociados a estos patógenos radica en los altos índices de resistencia a antimicrobianos que éstos presentan, limitando en gran medida las opciones terapéuticas y obligando al uso de antimicrobianos de mayor costo o incluso de última línea, como es el caso de polimixinas (colistín), al no existir otras alternativas de tratamiento dentro de los esquemas tradicionales ${ }^{6}$. Entre estas bacterias destacan Pseudomonas aeruginosa y Acinetobacter baumannii resistentes a carbapenémicos, así como cepas de Escherichia coli y Klebsiella pneumoniae productoras de $\beta$-lactamasas de espectro extendido (BLEE) ${ }^{7}$.

Debido a las limitadas alternativas para enfrentar este problema, la prevención adquiere especial relevancia. El cobre, gracias a sus propiedades bactericidas, se presenta como una alternativa para ser utilizado como recubrimiento de superficies, que habitualmente presentan una alta carga microbiana, en los recintos 
hospitalarios, especialmente aquellas denominadas "puntos de alto contacto" como barandas de camas, mesa de alimentación, bombas de infusión, etc. El cobre en bajas concentraciones es un micronutriente celular, pero en elevadas concentraciones posee actividad citotóxica, la que se debe principalmente a la inactivación competitiva de sistemas enzimáticos celulares ${ }^{8}$ y a su capacidad de producir especies radicales de oxígeno que dañan diversas estructuras de la bacteria ${ }^{9,10}$. Esta capacidad antibacteriana ha sido evidenciada en diversos estudios $^{11,12}$ e incluso la Agencia de Protección Ambiental de Estados Unidos (EPA) avaló el uso de aleaciones de cobre como material con propiedades antibacterianas ${ }^{13}$. Estudios realizados en Chile en el ámbito clínico han demostrado un notable efecto antibacteriano de superficies cobrizadas en unidades de cuidado intensivo, con disminuciones entre 49 y $92 \%$ de la carga bacteriana en las superficies evaluadas ${ }^{14}$.

No obstante lo anterior, se han descrito mecanismos de tolerancia a este metal en variados géneros bacterianos, dentro de los que se incluye bacilos gramnegativos de importancia clínica. Estos mecanismos pueden participar en la homeostasis natural del cobre o conferir propiedades adicionales de resistencia a estas bacterias, permitiéndoles soportar elevadas concentraciones del metal ${ }^{15,16}$. En general, ellos apuntan a la detoxificación de las formas iónicas del cobre tanto en el citoplasma como en el espacio periplásmico, involucrando bombas de expulsión, tanto ATPasas como transportadores dependientes del gradiente de protones, y enzimas oxidativas, que tendrían la función de alterar el estado de oxidación de los iones de cobre disminuyendo su toxicidad ${ }^{17}$. Sin embargo, hay evidencia de su contribución en el aumento de la supervivencia de las bacterias que los poseen en superficies metálicas ${ }^{18}$. Se han descrito eventos de co-selección de genes de resistencia, tanto a metales como a antimicrobianos, asociados generalmente a la presión selectiva de tan solo uno de ellos $^{19}$, lo que se explica, generalmente, por la presencia de ambos determinantes de resistencia en las mismas estructuras genéticas, como por ejemplo plásmidos. En el caso de la tolerancia bacteriana a cobre iónico, ésta ha sido descrita en asociación a genes de resistencia a antimicrobianos como por ejemplo cefalosporinas de tercera generación ${ }^{20}$ y glicopéptidos ${ }^{21}$.

La asociación de ambos tipos de resistencias en cepas patógenas sometidas a alta presión selectiva puede producir la co-selección de cepas multi-resistentes a antimicrobianos $\mathrm{y}$, a su vez, tolerantes a cobre en ambientes hospitalarios. En relación a lo anteriormente planteado el presente estudio investigó la actividad de cobre (en forma de sulfato cúprico, $\mathrm{CuSO}_{4}$ ) sobre bacilos gramnegativos hospitalarios productores y no productores de BLEE, y resistentes o susceptibles a antimicrobianos carbapenémicos.

\section{Material y Método}

Cepas bacterianas. Se analizó 390 cepas de bacilos gramnegativos aislados en 16 hospitales chilenos de las ciudades de Antofagasta, Valparaíso, Viña del Mar, Santiago, Constitución, San Carlos, Concepción, Talcahuano, Coronel, Lota, Temuco y Puerto Montt, entre los años 1997 y 2010, pertenecientes a la colección de cepas del Laboratorio de Investigación en Agentes Antibacterianos, Departamento de Microbiología, Facultad de Ciencias Biológicas de la Universidad de Concepción. Las cepas bacterianas correspondieron a A. baumannii (71), $P$. aeruginosa (99), K. pneumoniae (109) y E. coli (111). El criterio de selección fue susceptibilidad o resistencia a antimicrobianos carbapenémicos (imipenem y/o meropenem) para los bacilos gramnegativos no fermentadores, y presencia o ausencia de BLEE para las Enterobacteriaceae (Tablas 1 y 2). La producción de BLEE fue confirmada por el método fenotípico confirmatorio recomendado por el Clinical and Laboratory Standards Institute $^{22}$ y amplificación de los genes específicos por RPC. La resistencia a carbapenémicos en los bacilos gramnegativos no fermentadores fue confirmada determinando la concentración inhibitoria mínima (CIM) por dilución seriada en placas ${ }^{23}$. Las cepas fueron sembradas

Tabla 1. Distribución de CIM a sulfato cúprico en cepas de Enterobacteriaceae productoras y no productoras de BLEE

\begin{tabular}{|lccccccc|}
$\begin{array}{l}\text { Especie } \\
\text { bacteriana }\end{array}$ & Fenotipo & $\mathbf{n}$ cepas & $\begin{array}{c}\text { Rango } \\
\mathrm{CIM}\end{array}$ & $\begin{array}{c}\mathrm{CIM}_{50} \\
(\mathrm{mM})\end{array}$ & $\begin{array}{c}\mathrm{CIM}_{90} \\
(\mathrm{mM})\end{array}$ & $\begin{array}{c}\mathbf{M G}_{\mathrm{cIM}} \\
(\mathrm{mM})^{\dagger}\end{array}$ & Valor $\mathbf{p}$ \\
K. pneumoniae & $\mathrm{BLEE}^{+*}$ & 53 & $8-10$ & 10 & 10 & 9,132 & $<0,001^{++}$ \\
& $\mathrm{BLEE}^{-* *}$ & 56 & $6-10$ & 6 & 10 & 7,345 & \\
E. coli & $\mathrm{BLEE}^{+}$ & 57 & 6 & 6 & 6 & 6,000 & NA \\
& $\mathrm{BLEE}^{-}$ & 54 & 6 & 6 & 6 & 6,000 & \\
\hline
\end{tabular}

${ }^{*} \mathrm{BLEE}^{+}$: cepas productoras de $\beta$-lactamasas de espectro extendido; ${ }^{*}{ }^{*} \mathrm{BLEE}^{-}$: cepas no productoras de $\beta$-lactamasas de espectro extendido; ${ }^{\top} \mathrm{MG}_{\mathrm{CMI}}$ : media geométrica de las $\mathrm{CIM}$ de CuSO4; ${ }^{\dagger+}$ Estadísticamente significativo; NA: no aplicado.

Tabla 2. Distribución de CIM a sulfato cúprico en cepas de bacilos gramnegativos no fermentadores resistentes y susceptibles a antimicrobianos carbapenémicos

\begin{tabular}{|c|c|c|c|c|c|c|c|}
\hline $\begin{array}{l}\text { Especie } \\
\text { bacteriana }\end{array}$ & Fenotipo & n cepas & $\begin{array}{c}\text { Rango } \\
\text { CIM } \\
(\mathrm{mM})\end{array}$ & $\begin{array}{l}\mathrm{CIM}_{50} \\
(\mathrm{mM})\end{array}$ & $\begin{array}{l}\mathrm{CIM}_{90} \\
(\mathrm{mM})\end{array}$ & $\begin{array}{l}\mathbf{M G}_{\mathrm{cIM}} \\
(\mathrm{mM})^{\dagger}\end{array}$ & Valor $p$ \\
\hline \multirow[t]{2}{*}{ A. baumannii } & $\mathrm{CAR}^{R *}$ & 35 & $8-10$ & 10 & 10 & 9,714 & \multirow{2}{*}{$<0,001^{\text {th }}$} \\
\hline & $\mathrm{CAR}^{5 * *}$ & 36 & $1-10$ & 6 & 8 & 5,361 & \\
\hline \multirow[t]{2}{*}{$P$. aeruginosa } & $\mathrm{CAR}^{\mathrm{R}}$ & 50 & $8-10$ & 8 & 10 & 8,680 & \multirow{2}{*}{0,148} \\
\hline & $C A R^{S}$ & 49 & $2-10$ & 8 & 10 & 8,296 & \\
\hline
\end{tabular}

${ }^{*} C A R^{R}$ : cepas resistentes a carbapenémicos; ${ }^{* *} C A R^{5}$ : cepas susceptibles a carbapenémicos; ${ }^{\dagger} \mathrm{MG}_{\mathrm{CMI}}$ : media geométrica de la CIM a CuSO4; "HEstadísticamente significativo. 
desde el cepario (donde se conservaban en glicerol 50\% $\mathrm{v} / \mathrm{v}$ y caldo de cultivo, a $-80^{\circ} \mathrm{C}$ ) en agar Mueller-Hinton (Oxoid, Inglaterra) con discos de antimicrobianos como presión selectiva, previo a su utilización en los experimentos realizados. Como controles se utilizó las cepas E. coli ATCC 25922 y P. aeruginosa ATCC 27853.

Estudio de susceptibilidad a agentes antibacterianos. Este estudio se realizó mediante el método de difusión en agar Mueller-Hinton (Oxoid), siguiendo las recomendaciones y puntos de corte propuestos por el CLSI ${ }^{24}$. Los antimicrobianos y la potencia de los discos utilizados fueron: gentamicina (GEN, $10 \mu \mathrm{g}$ ), amikacina (AMK, $30 \mu \mathrm{g})$, ceftriaxona (CRO, $30 \mu \mathrm{g})$, ceftazidima (CAZ, 30 $\mu \mathrm{g})$, cefotaxima (CTX, $30 \mu \mathrm{g})$, cefepima (FEP, $30 \mu \mathrm{g})$, imipenem (IPM, $10 \mu \mathrm{g}$ ), meropenem (MEM, $10 \mu \mathrm{g}$ ), ertapenem (ETP, $10 \mu \mathrm{g})$.

Actividad antibacteriana de $\mathrm{CuSO}_{4} \mathrm{La}$ CIM de cobre se determinó mediante el método de dilución seriada en placas de agar Mueller-Hinton (Oxoid) utilizando $\mathrm{CuSO}_{4}$ (Merck). Para ello se utilizó un inóculo bacteriano final de $10^{4}$ ufc por cepa (obtenido mediante turbidometría), aplicado mediante un replicador Steers, en placas de agar Mueller-Hinton adicionadas de $\mathrm{CuSO}_{4}$ en las siguientes concentraciones: 1, 2, 4, 6, 8, 10, 12, 16 y $32 \mathrm{mM}$, se incubó a $37^{\circ} \mathrm{C}$ por $48 \mathrm{~h}$ y se determinó la CIM de $\mathrm{CuSO}_{4}$.

Análisis estadístico. La significancia estadística de las diferencias de las medias geométricas de los niveles de tolerancia a cobre entre los distintos grupos de cepas de las especies estudiadas (cepas resistentes o susceptibles a

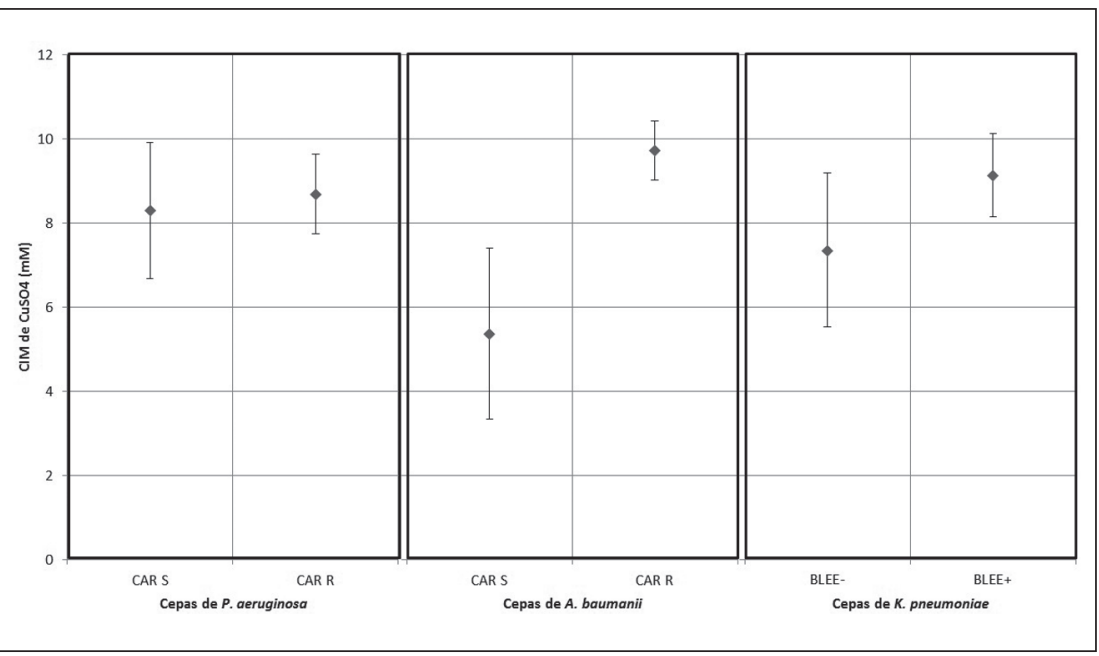

Figura 1. Medias geométricas y desviación estándar de la CIM a sulfato cúprico de cepas de $P$. aeruginosa, A. baumannii y K. pneumoniae. CAR S: cepas susceptibles a carbapenémicos, CAR R: cepas resistentes a carbapenémicos, BLEE-: cepas no productoras de $\beta$-lactamasas de espectro extendido, $\mathrm{BLEE}+$ : cepas productoras de $\beta$-lactamasas de espectro extendido. antimicrobianos carbapenémicos para $P$. aeruginosa y $A$. baumannii; cepas productoras o no productoras de BLEE para K. pneumoniae y E. coli), fue determinada mediante la prueba t de Student desapareada con dos colas ${ }^{25}$. Se consideró estadísticamente significativo un $\mathrm{p}<0,05$ con un intervalo de confianza de $95 \%$.

\section{Resultados}

\section{Susceptibilidad antimicrobiana de las cepas}

Las cepas de K. pneumoniae y E. coli no productoras de BLEE (BLEE-) presentaron un porcentaje elevado de susceptibilidad a la totalidad de los antimicrobianos ensayados, mientras que entre aquellas productoras de BLEE $\left(\mathrm{BLEE}^{+}\right)$se encontró un elevado porcentaje de cepas resistentes a todas las cefalosporinas ensayadas (CRO, CAZ, CTX y FEP), pero presentando un alto porcentaje de susceptibilidad a carbapenémicos. Sin embargo, entre las cepas de K. pneumoniae productoras de BLEE se observó un menor porcentaje de cepas susceptibles a ertapenem. En las cepas de $P$. aeruginosa resistentes a antimicrobianos carbapenémicos $\left(\mathrm{CAR}^{\mathrm{R}}\right)$ se observó un mayor número de cepas resistentes a cefalosporinas de tercera generación (CRO, CAZ CTX) con respecto a las cepas susceptibles a carbapenémicos $\left(\mathrm{CAR}^{\mathrm{S}}\right)$; sin embargo, esta diferencia no se detectó para los aminoglucósidos (GEN y AMK), ni tampoco para la cefalosporina de cuarta generación cefepima. Las cepas de $A$. baumannii $\mathrm{CAR}^{\mathrm{R}}$ presentaron perfiles de multi-resistencia que incluyó a todos los antimicrobianos ensayados, salvo en un bajo porcentaje de cepas que fueron susceptibles a gentamicina (28\%).

\section{Actividad antibacteriana de $\mathrm{CuSO}_{4}$}

Se observaron mayores niveles de tolerancia a cobre iónico en las cepas de $K$. pneumoniae $\mathrm{BLEE}^{+}$y $A$. baumannii $\mathrm{CAR}^{\mathrm{R}}$ que en sus contrapartes no productoras de BLEE y susceptibles a carbapenémicos, respectivamente.

Las cepas de $P$. aeruginosa analizadas presentaron niveles de tolerancia similares sin importar sus perfiles de resistencia a antimicrobianos (Figura 1). Un fenómeno similar fue observado en los grupos de cepas de $E$. coli estudiados. $\mathrm{La} \mathrm{CIM}_{50}$ de $\mathrm{CuSO}_{4}$ para cepas de A. baumannii $\mathrm{CAR}^{\mathrm{R}}$ y K. pneumoniae $\mathrm{BLEE}^{+}$fue $10 \mathrm{mM}$; mientras que para las las contrapartes $\mathrm{CAR}^{\mathrm{S}}$ y $\mathrm{BLEE}^{-}$fue $6 \mathrm{mM}$. En $P$. aeruginosa no se observó diferencias de la $\mathrm{CIM}_{50}$ de $\mathrm{CuSO}_{4}$ entre $\mathrm{CAR}^{\mathrm{R}}$ y CAR ${ }^{\mathrm{S}}$, siendo para ambas $8 \mathrm{mM}$. Estos resultados se muestran en las Tablas 1 y 2 .

Análisis estadístico. Los análisis realizados para determinar la significancia estadística de las diferencias de las medias geométricas de las CIM a $\mathrm{CuSO}_{4}$ entre los grupos de cepas, indicaron valores de $\mathrm{p}<0,001$ para $K$. pneumoniae y $A$. baumannii y de $\mathrm{p}=0,148$ para $P$. aeru- 
ginosa. Debido a que la totalidad de las cepas de $E$. coli presentaron niveles de tolerancia similares, no se realizó el análisis para esta especie.

\section{Discusión}

El análisis estadístico de los niveles de tolerancia a cobre determinados en este estudio demostró una diferencia estadísticamente significativa, considerando un intervalo de confianza de $95 \%$, entre las medias geométricas de las $\mathrm{CIM}$ de $\mathrm{CuSO}_{4}$ de las cepas de $A$. baumannii resistentes y susceptibles a antimicrobianos carbapenémicos $(\mathrm{p}<0,001)$; así como entre las cepas de K. pneumoniae productoras y no productoras de BLEE $(\mathrm{p}<0,001)$. En ambos casos las cepas con mayores niveles de resistencia a antimicrobianos $\left(\mathrm{CAR}^{\mathrm{R}}\right.$ y $\mathrm{BLEE}^{+}$, respectivamente) presentaron mayores niveles de tolerancia a cobre iónico. En las cepas de $P$. aeruginosa no se observó esta relación, ya que la mayoría de las cepas, tanto resistentes como susceptibles a carbapenémicos, presentaron niveles similares de tolerancia a cobre $(\mathrm{p}=0,148)$. En $A$. baumannii y $P$. aeruginosa es esperable encontrar mayores niveles de resistencia a metales debido a su intrínseca impermeabilidad de membrana que favorece la resistencia a diversos agentes antibacterianos ${ }^{4}$; sin embargo, los mecanismos moleculares responsables de la menor susceptibilidad a cobre en las cepas de $A$. baumannii $\mathrm{CAR}^{\mathrm{R}}$, así como los observados en las cepas de $K$. pneumoniae $\mathrm{BLEE}^{+}$, aún deben ser dilucidados. Los niveles de tolerancia a cobre en cepas de E. coli no mostraron diferencias entre las cepas productoras y no productoras de BLEE y se correlacionaron, además con el nivel de tolerancia a cobre observado con la cepa control E. coli ATCC 25922, sugiriendo la ausencia de mecanismos adicionales de tolerancia a cobre en esta especie.

Se han descrito diversos sistemas moleculares que podrían favorecer la co-selección de determinantes de tolerancia a cobre junto con genes de resistencia a antimicrobianos en bacilos gramnegativos patógenos. En $P$. aeruginosa se describió un sistema de dos componentes (CopR-CopS) activado por cobre y que se ha implicado en la tolerancia a este metal, así como en la activación de mecanismos de tolerancia a zinc y la pérdida de la porina OprD, involucrada en la captación de imipenem, produciendo de este modo resistencia a este antimicrobiano ${ }^{26}$. Este fenómeno también ha sido descrito en asociación con elementos genéticos móviles, como el plásmido conjugativo pK29 de K. pneumoniae, en el cual residen genes que codifican $\beta$-lactamasas del tipo CTX-M (BLEE) y CMY (AmpC plasmídica) junto con genes relacionados con tolerancia a cobre, además de otros genes de resistencia a metales y antimicrobianos ${ }^{20}$. Por otra parte, estudios realizados en bacterias grampositivas han mostrado re- sultados similares. Un ejemplo es el informado para cepas de Enterococcus faecium que presentaron fenotipos de resistencia transferibles a antimicrobianos glicopeptídicos, macrólidos y a cobre en un mismo evento de transferencia horizontal de genes $^{21}$. La evidencia experimental señala la posibilidad de co-selección de cepas con fenotipos de multi-resistencia a antimicrobianos asociados a la resistencia a metales ${ }^{19}$. Los resultados obtenidos en este estudio permiten observar que el caso de la tolerancia a cobre iónico no es una excepción de este fenómeno. Las diferencias observadas entre los niveles de tolerancia a cobre iónico y su relación con la resistencia a ciertos antimicrobianos en las distintas especies estudiadas podrían ser atribuidas a las características particulares de cada una; sin embargo, debe profundizarse la investigación de este fenómeno para poder dar una explicación concreta de estas asociaciones.

Los mecanismos moleculares de tolerancia a cobre iónico han demostrado tener incidencia en la capacidad de supervivencia bacteriana en superficies cobrizadas, donde la deleción de genes cromosómicos ${ }^{27,28}$ o la presencia de genes de origen plasmídicos ${ }^{18}$ de tolerancia al metal demostraron alterar el tiempo de sobrevida de las bacterias. Cabe destacar que esto no aseguraría una sobrevida a largo plazo, pero podría favorecer la selección de aquellas cepas que presenten estos mecanismos. No obstante, esto debe ser estudiado de manera más profunda, ya que los mecanismos de toxicidad del cobre metálico, aunque se ha visto que involucran la liberación de iones de cobre, no han sido esclarecidos a cabalidad ${ }^{17}$. Los resultados obtenidos en este estudio permiten observar una relación entre la disminución de la susceptibilidad a cobre iónico y a antimicrobianos de última generación en $K$. pneumoniae y A. baumannii. Sin embargo, se deben realizar estudios adicionales para establecer los mecanismos moleculares involucrados en esta tolerancia, así como la capacidad de transferencia de ellos. Aunque en este trabajo no se utilizaron superficies cobrizadas, existe evidencia que la muerte bacteriana se produce por liberación de cobre iónico ${ }^{18}$, por lo que sería de mucho interés estudiar la supervivencia de estas cepas bacterianas en superficies de cobre. En su conjunto, esto permitiría obtener información acerca de la existencia de un potencial riesgo de selección de cepas de bacilos gramnegativos productor de BLEE y/o resistente a carbapenémicos debido al uso de superficies cobrizadas en los hospitales. Esto es de vital importancia si se considera la situación epidemiológica de nuestros hospitales y las limitadas opciones terapéuticas, por lo que resulta imperioso dilucidar el efecto que podría tener la implementación de superficies cobrizadas sobre la selección de bacterias multi-resistentes, y así utilizar de manera segura una medida de control de microrganismos hospitalarios que tiene un elevado costo de implementación. 
Agradecimientos. A la Facultad de Ciencias Biológicas de la Universidad de Concepción por el financiamiento entregado para la realización de este estudio. A los microbiólogos de los siguientes hospitales que proporcionaron las cepas incluidas en este estudio: "Dr. Leonardo Guzmán” de Antofagasta; Naval “Almirante Nef" de Viña del Mar; Carlos Van Buren de Valparaíso; Clínico de la Pontificia Universidad Católica de Chile de Santiago; Clínico de la Universidad de Chile "Dr. José Joaquín Aguirre" de Santiago; Barros Luco-Trudeau de Santiago; Clínico San Borja-Arriarán de Santiago; San Juan de Dios de Santiago; San José de Santiago; Complejo Asistencial "Dr. Sótero del Río" de Santiago; Hospital de Constitución (Maule); Hospital de San Carlos (San Carlos, Bío Bío); "Dr. Guillermo Grant B.” de Concepción; Las Higueras de Talcahuano; Naval "Almirante Adriazola" de Talcahuano; San José de Coronel; Hospital de Lota; "Dr. Hernán Henríquez Aravena" de Temuco y Hospital de Puerto Montt.

\section{Resumen}

Introducción: Los patógenos intrahospitalarios multiresistentes constituyen un grave problema mundial de salud pública, especialmente los bacilos gramnegativos (BGN). El uso de cobre como antimicrobiano de superficie en hospitales se postula como una alternativa para el control de microorganismos en estos ambientes. Sin embargo, la multi-resistencia a antimicrobianos en BGN hospitalarios puede asociarse con la tolerancia a cobre, ya que existe evidencia que genes que codifican tolerancia a este metal pueden encontrarse en elementos genéticos que confieren resistencia a antimicrobianos. Por esta razón, cepas multi-resistentes a antimicrobianos podrían sobrevivir en presencia de cobre, seleccionando bacterias resistentes a ambos agentes antibacterianos. Objetivo: Investigar la actividad de cobre sobre BGN hospitalarios productores y no productores de $\beta$-lactamasas de espectro extendido (BLEE), y resistentes o susceptibles a antimicrobianos carbapenémicos. Material y Métodos: Se estudió 390 cepas de BGN aisladas en hospitales chilenos: Acinetobacter baumannii y Pseudomonas aeruginosa resistentes $\left(\mathrm{CAR}^{\mathrm{R}}\right)$ y susceptibles $\left(\mathrm{CAR}^{\mathrm{S}}\right)$ a carbapenémicos y Klebsiella pneumoniae y Escherichia coli productoras y no productoras de BLEE. Se investigó los niveles de susceptibilidad a sulfato cúprico, mediante dilución seriada en agar y se evaluó la significancia estadística de la diferencia de estos niveles entre los distintos grupos de cepas. Resultados: Se encontraron niveles de tolerancia a cobre superiores en cepas de $A$. baumannii y $K$. pneumoniae, $\mathrm{CAR}^{\mathrm{R}}$ y productoras de BLEE respectivamente, con respecto a sus pares $\mathrm{CAR}^{\mathrm{S}}$ y no productoras de BLEE. Conclusión: Observamos una relación entre la disminución de la susceptibilidad a cobre iónico y a antimicrobianos de última generación en K. pneumoniae y A. baumannii.

\section{Referencias bibliográficas}

1.- López-Pueyo M, Barcenilla-Gaite F, Amaya-Villar R y Garnacho-Montero J. Multirresistencia antibiótica en unidades de críticos. Med Intensiva 2011; 35: 41-53.

2.- Brenner P, Nercelles P, Pohlenz M, Otaíza F. Costo de las infecciones intrahospitalarias en hospitales chilenos de alta y mediana complejidad. Rev Chilena Infectol 2003; 20 : 285-90.

3.- Boum J, Ashby S, Sedgwick J, Pearson A, Taylor K. Improving patient care by reducing the risk of hospital-acquired infection: a progress report. National Audit Office 2004. http://www.nao.org.uk/publications/0304/ improving_patient_care.aspx. Octubre 2011.

4.- Rice L. Progress and challenges in implementing the research on ESKAPE pathogens. Infect Control Hosp Epidemiol 2010; 31: S7-S10.

5.- Otaíza F, Pohlenz M, Brenner P, Bustamante R. Informe de la vigilancia de infecciones intrahospitalarias. Ministerio de Salud de Chile 2005.

6.- Levy S. Antibiotic resistance - the problem intensifies. Adv Drug Deliv Rev 2005; 57 : 1446-50.
7.- Slama T. Gram-negative antibiotic resistance: there is a price to pay. Crit Care 2008; 12: S4.

8.- Macomber L, Imlay J. The iron-sulfur clusters of dehydratases are primary intracellular targets of copper toxicity. PNAS 2009; 106 : 8344-9.

9.- Halliwell B, Gutteridge J. Oxygen toxicity, oxygen radicals, transition metals and disease. Biochem J 1984; 219: 1-14.

10.- Avery S, Howlett N, Radice S. Copper toxicity towards Saccharomyces cerevisiae: dependence on plasma membrane fatty acid composition. Appl Environ Microbiol 1996; 62: 3960-6.

11.- Noyce J, Michels H, Keevil C. Potential use of copper surfaces to reduce survival of epidemic methicillin-resistant Staphylococcus aureus in the healthcare environment. J Hosp Infect 2006; 63: 289-97.

12.- Mehtar S, Wiid I, Todorov S. The antimicrobial activity of copper and copper alloys against nosocomial pathogens and Mycobacterium tuberculosis isolated from healthcare facilities in the Western Cape: an in-vitro study. J Hosp Infect 2008; 68: 45-51.

13.- EPA registers copper-containing alloy products http://www.epa.gov/pesticides/factsheets/ copper-alloy-products.htm, Enero 2012.
14.- Prado V, Durán C, Crestto M, Gutiérrez A, Sapiain P, Flores G, et al. Effectiveness of copper contact surfaces in reducing the microbial burden (MB) in the intensive care unit (ICU) of Hospital del Cobre, Calama, Chile. 2010. http://www.findthatpdf.com/search7918642-hPDF/download-documents-ificposter-calama-results.pdf.htm.diciembre 2012 (accedido enero 2012).

15.- Rosen B. Transport and detoxification systems for transition metals, heavy metals and metalloids in eukaryotic and prokaryotic microbes. Comp Biochem Physiol A Mol Integr Physiol 2002; A133: 689-93.

16.- Boal A, Rosenzweig A. Structural biology of copper trafficking. Chem Rev 2009; 109: 4760-79.

17.- Grass G, Rensing C, Solioz M. Metallic copper as an antimicrobial surface. App Environ Microbiol. 2011; 77: 1541-7.

18.- Espírito Santo C, Taudte N, Nies D, Grass G. Contribution of copper ion resistance to survival of Escherichia coli on metallic copper surfaces. Appl Environ Microbiol 2008; 74: 977-86.

19.- Baker-Austin C, Wright M, Stepanauskas R y McArthur J. Co-selection of antibiotic and metal resistance. Trends Microbiol 2006; 14: 176-82.

20.- Chen Y, Lauderdale T, Liao T, Shiau Y, 
Shu $\mathrm{H}, \mathrm{Wu} \mathrm{K}$ et al. Sequencing and comparative genomic analysis of $\mathrm{pK} 29$, a 269-kilobase conjugative plasmid encoding CMY-8 and CTX-M-3 $\beta$-lactamases in Klebsiella pneumoniae. Antimicrob Agents Chemother 2007; 51: 3004-7.

21.- Hasman H, Aarestrup F. tcrB, a gene conferring transferable copper resistance in Enterococcus faecium: occurrence, transferability, and linkage to macrolide and glycopeptide resistance. Antimicrob Agents Chemother 2002; 46: 1410-6.

22.- Clinical and Laboratory Standards Institute. Performance standards for antimicrobial susceptibility testing; Twentieth Informational Supplement. CLSI document M100-S20. (ISBN 1-56238-716-2). Clinical and Laboratory
Standards Institute, Wayne, Pennsylvania, USA, 2010.

23.- Clinical and Laboratory Standards Institute. Methods for Dilution Antimicrobial Susceptibility Tests for Bacteria That Grow Aerobically; Approved Standard - Ninth Edition. CLSI document M07-A9. (ISBN 1-56238-783-9). Clinical and Laboratory Standards Institute, Wayne, Pennsylvania, USA, 2010.

24.- Clinical and Laboratory Standards Institute. Performance Standards for Antimicrobial Disk Susceptibility Tests; Approved Standard - Eleventh Edition. CLSI document M02-A11. Clinical and Laboratory Standards Institute, Wayne, Pennsylvania, USA, 2010.
25.- De Muth JE. Basic statistics and pharmaceutical statistical applications. 2nd ed. Boca Raton: Chapman and Hall/CRC; 2006.

26.- Caille O, Possier C, Perron K. A copperactivated two-component system interacts with zinc and imipenem resistance in Pseudomonas aeruginosa. J Bacteriol 2007; 189: 4561-8.

27.- Elguindi J, Wagner J y Rensing C. Genes involved in copper resistance influence survival of Pseudomonas aeruginosa on copper surfaces. J Appl Microbiol 2009; 106: 1448-55.

28.- Molteni C, Abicht H, Solioz M. Killing of bacteria by copper surfaces involves dissolved copper. Appl Environ Microbiol 2010; 76: 4099-101. 